\title{
Neural Networks That Distinguish Infection Periods of Wheat Tan Spot in an Outdoor Environment
}

\author{
E. D. De Wolf and L. J. Francl
}

North Dakota State University, Department of Plant Pathology, Fargo 58105.

Accepted for publication 16 September 1996.

\begin{abstract}
De Wolf, E. D., and Francl, L. J. 1997. Neural networks that distinguish infection periods of wheat tan spot in an outdoor environment. Phytopathology 87:83-87.

Tan spot of wheat, caused by Pyrenophora tritici-repentis, provided a model system for testing disease forecasts based on an artificial neural network. Infection periods for $P$. tritici-repentis on susceptible wheat cultivars were identified from a bioassay system that correlated tan spot incidence with crop growth stage and 24-h summaries of environmental data, including temperature, relative humidity, wind speed, wind direction, solar radiation, precipitation, and flat-plate resistance-type wetness sensors. The resulting data set consisted of 97 discrete periods, of which 32 were reserved for validation analysis. Neural networks with zero to

nine processing elements were evaluated 20 times each to identify the model that most accurately predicted an infection event. The 200 models averaged 74 to $77 \%$ accuracy, depending on the number of processing elements and random initialization of coefficients. The most accurate model had five processing elements and correctly predicted $87 \%$ of the infection periods in the validation set. In comparison, stepwise logistic regression correctly predicted $69 \%$ of the validation cases, and multivariate discriminant analysis distinguished $50 \%$ of the validation cases. When wetness-sensor inputs were withheld from the models, both the neural network and logistic regression models declined 6\% in prediction accuracy. Thus, neural networks were more accurate than statistical procedures, both with and without wetness-sensor inputs. These results demonstrate the applicability of neural networks to plant disease forecasting.
\end{abstract}

Plant disease forecasts assist growers in timing disease management activities and can improve farm production efficiency while reducing pesticide applications (1). An economical approach to plant disease forecasting assumes the pathogen is present and bases forecasts on environmental variables that can be recorded automatically. In general, these forecasting systems attempt to define how various components of disease cycles (infection, sporulation, latency, etc.) interact with sporadic and discontinuous environmental factors. Although experiments to quantify these interactions traditionally have been done in greenhouses or growth chambers, verification in outdoor environments is highly desirable, despite the sacrifice of environmental control $(5,15,16)$.

Spring wheat, Triticum aestivum L., and durum wheat, T. turgidum var. durum Desf., are the predominant cultivated wheat crops of the northern Great Plains, and many common wheat cultivars are susceptible to foliar diseases. Tan spot, caused by Pyrenophora tritici-repentis (Died.) Drechs., is the most common wheat leaf disease in North Dakota (11). Tan spot is endemic throughout the Great Plains and in many other semiarid regions where wheat is grown intensively $(3,10,13)$. Various components of the disease cycle have been studied, both in controlled and outdoor environments $(5,6,8,13)$. Both ascospores and conidia are infectious propagules of the pathogen, but conidia are of primary importance during later growth stages of wheat when the crop is most at risk of damage (13). The importance of tan spot, the potential economic return of a single well-timed fungicide application, and knowledge of the relationships between various environmental events and tan spot infection provide an excellent model system for testing plant disease forecasting.

Artificial neural network (ANN) technology was developed conceptually in the 1940s and 1950s. Recently, new architectures

Corresponding author: E. D. De Wolf; E-mail address: edewolf@plains.nodak.edu

Publication no. P-1996-1018-01R

(C) 1997 The American Phytopathological Society have fueled a resurgence of interest in the technology $(17,19)$. ANN applications in the agricultural sciences have been researched for a wide range of classification, optimization, and prediction problems $(2,4,12,18)$. Application research in plant pathology, so far, includes the prediction of leaf wetness duration (7) and soybean rust progress (20).

An ANN is similar to multivariate nonlinear regression analysis in that both evaluate independent variables and iteratively develop coefficients that model patterns within the data set with a minimal error. However, statistical models, whether linear or nonlinear, must have a formal solution specified initially by the modeler. With an ANN model, how information is to be processed can be specified at the outset, but multistep, parallel, and interconnected processing during thousands of iterations determines the final form of the model. Many different ANN architectures have been developed and investigated to analyze patterns in data; the back-propagation technique has been used successfully to model many systems (17).

A feed-forward back-propagation model typically consists of at least three layers of connected processing elements (PEs). The network includes an input layer, one or more processing layers, and an output layer. Each input and output variable in the problem set is assigned a PE. In a fully connected network, each input PE has a processing path to every output PE through a user-selectable number of intermediate PEs. Information is fed forward to the output layer from the input layer. To detail the processing opera tion further, input PEs transfer values of independent variables to the PEs of the processing layer. Each PE of the processing layer calculates the weighted sum of the input values by unique con nection weights and performs a transfer function on the weighted sum. This transformed value is passed to the PEs of the next layer, which may be another processing layer or the output layer. Each PE of the output layer performs calculations similar to the PEs of the processing layer, and the resulting value is an estimate of the dependent variable. Back-propagation networks adjust each of the connection weights in a direction and magnitude that minimizes error between the estimated and actual outputs, and the process is repeated (17). 
An ANN model usually is presented with training cases in random order until the model reaches a preset criterion, such as an error threshold or classification accuracy. The model then can be validated by disabling the weight adjustment capabilities of the model and providing an independent set of variables. Thus, preexisting weights established during model development estimate the actual output values of the new data set. Modifications in the initial model states are tested by trial and error to find the model that minimizes error in the desired criterion $(17,19)$.

Our objective was to use a tan spot infection period as a model to find out how successfully ANN technology can be applied to plant disease forecasting. Our forecast models were based solely on crop growth stage and environmental variables collected by an automated weather station. The ANN model results were com- pared with the statistical models to evaluate ANN model performance. This approach tested the applicability of ANN technology for plant disease forecasting.

\section{MATERIALS AND METHODS}

Infection-period bioassays were conducted at the North Dakota State University Agricultural Experiment Station, Fargo, during June and July of the 1993, 1994, and 1995 growing seasons. Each year, a 1.0-ha field of the tan spot-susceptible wheat cv. Grandin was planted in a field cropped previously to wheat, with stubble left on the soil surface. Tan spot-susceptible hard red spring wheat line ND495 was planted weekly in 20-cm-diameter clay pots in a greenhouse. Plants were thinned to three plants per pot, fertilized

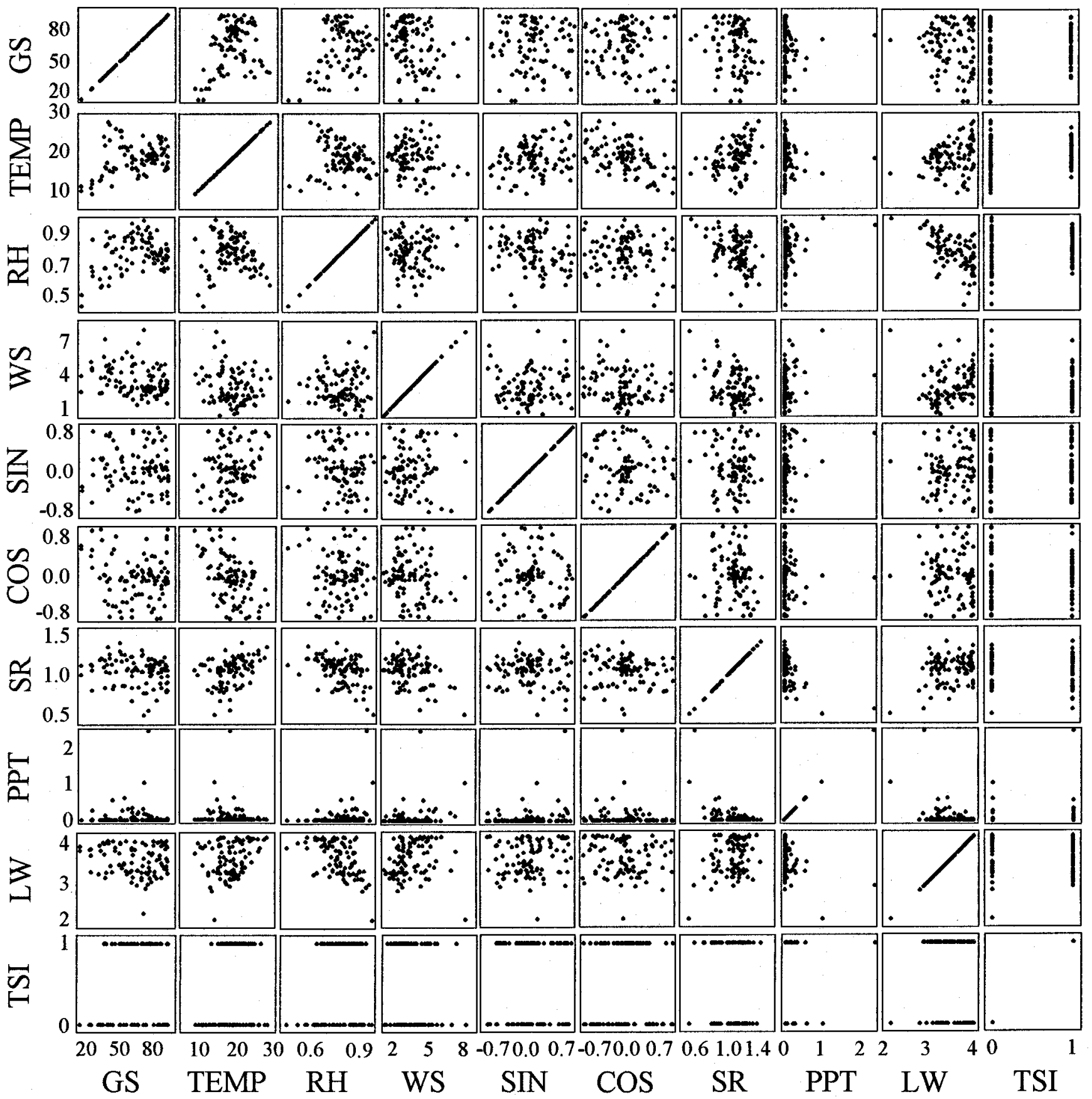

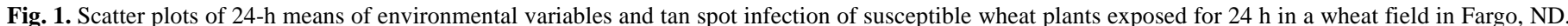

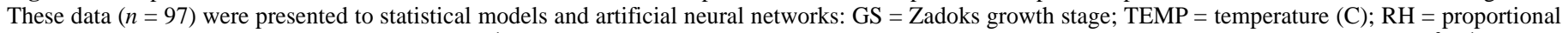

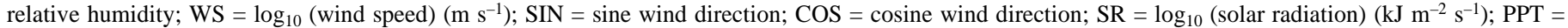
precipitation $(\mathrm{mm}) ; \mathrm{LW}=\log _{10}($ wetness sensor resistance $)(\mathrm{k} \Omega)$; TSI $=$ tan spot infection $(1=$ infection, $0=$ no infection $)$. 
with a time-release, complete fertilizer, and allowed to grow until the main tillers had reached the anthesis or early milk stage of growth. Infection-period bioassays began when the wheat field was in Zadoks (21) growth stage 13 (1993), 34 (1994), or 38 (1995) and continued until growth stage 93 (1993), 93 (1994), or 92 (1995). Two pots of wheat plants were exposed in the wheat field for 24-h periods beginning and ending at 10:00 a.m., after any dew had evaporated.

Exposed potted plants were transferred directly to a growth chamber, where they were paired with control plants left in a greenhouse. One of the two field-exposed plants and a control plant underwent a 24-h wet period in a mist chamber within the growth chamber, while the other field-exposed plant and a second control plant remained dry. The environment in the growth chamber was maintained at $21 \pm 2{ }^{\circ} \mathrm{C}$ with a 16 -h photoperiod $\left(400 \mu \mathrm{mol} \mathrm{s}{ }^{-1} \mathrm{~m}^{-2}\right)$. All plants in the growth chamber were irrigated from the bottom to avoid additional leaf wetness. After 10 days, plants were removed from the growth chamber, and the uppermost three leaves of two arbitrarily selected main tillers were inspected for the presence of visible lesions. Leaf sections bearing lesions were surfacesterilized in a $1.0 \% \mathrm{NaOCl}$ solution, placed on $1.5 \%$ water agar, and incubated at $21^{\circ} \mathrm{C}$ for approximately 14 days with alternating $12 \mathrm{~h}$ of light and dark. The leaves were inspected for $P$. triticirepentis conidiophores and conidia or pseudothecia and ascospores. Although other pathogens were observed (5), data for this analysis were limited to the incidence of $P$. tritici-repentis leaf infections ( $\tan$ spot infection $=1$; no infection $=0)$ of field-exposed plants that remained dry.

Weather in the wheat field was monitored continuously with a CR10 data logger (Campbell Scientific, Logan, UT) equipped with temperature, relative humidity $(\mathrm{RH})$, wind speed, wind direction, solar radiation, precipitation, and flat-plate resistance-type wetness sensors. Three wetness sensors faced south at a 45-degree angle and were positioned $0.2 \mathrm{~m}$ above the crop. The growth stage (21) of the wheat plots was monitored at intervals during the growing season, and foliar disease severity was measured between stages 72 and 77. A Burkard spore sampler estimated the amount of inoculum present in the same field and provided additional information about the effectiveness of the bioassay.

ANN model development. NeuralWorks Professional II Plus (NeuralWare, Inc., Pittsburgh) software installed on a PC can implement several network architectures, including back-propagation. In a preliminary analysis, various combinations of input data, transformations, model architectures, PE number, and transfer functions were evaluated for their influence on forecast performance (data not shown).

Output data consisted of 97 cases of tan spot infection or noninfection on bioassay plants. Environmental data included 24-h averages of temperature (degrees Celsius), RH (percent), wind speed (meters per second), wind direction (degrees), solar radiation (kilojoules per square meter per second), precipitation (millimeters), and flat-plate wetness-sensor resistance (kiloohms). Due to the nature of some of the environmental inputs and the sensitivity of models to input data distribution, some transformations were deemed necessary (Fig. 1). Wind direction was transformed into sine and cosine values, because wind direction measurements are continuously circular. RH was rescaled between zero and one to limit the magnitude of the values. The $\log _{10}(x)$ of solar radiation and flat-plate resistance were used to avoid coefficient saturation. (Coefficient saturation occurs when changes in the coefficient of a variable or group of variables during successive iterations of a neural network fails to produce a meaningful change in the weight of one or more linked PEs.) Solar radiation data were unique, because the data set contained values $>10^{3}$ during the day, values $<1$ for periods near dust or dawn, and values of $0 \mathrm{~kJ} \mathrm{~m}^{-2} \mathrm{~s}^{-1}$ at night, making $\log _{10}(x)$ transformation impossible. To solve this problem, night readings were set to a decimal less than the dusk and dawn values (0.001), which produced a nearly symmetric distribution of the data about zero after transformation.
To construct the ANN and statistical models, 65 of the cases were picked arbitrarily by removing every third case. The other 32 cases were reserved for validation of the models. This method of selection resulted in an even distribution of cases within years and growth stages but an approximate randomization of other variables due to the nature of weather. Because many automated weather stations are not equipped with leaf-wetness sensors, ANN and statistical models were constructed both with and without the wetness-sensor input.

Multivariate discriminant analysis and stepwise logistic regression (SAS Institute, Inc., Cary, NC) processed the same training and validation data sets as the ANNs. Both of these techniques are recognized as the analytical methods of choice for categorization of a binary response (9; SAS). The discriminant analysis distinguished infection and noninfection periods by a parametric discriminant function based on within-group covariance matrices. The full set of input variables determined the discriminant function. Logistic regression fits a symmetrical S-shaped curve to binary data. The most significant independent variable was added stepwise to the logistic model in a forward-selection, backward-elimination process. All models were evaluated for accuracy based on the midpoint between zero and one.

Initialization of ANN coefficients with a random number resulted in variable model results. To compensate for this variation, 20 replicate ANNs with zero to nine PEs in the processing layer were evaluated for prediction accuracy (correct predictions per total number of cases) with respect to tan spot infection events. During the iterative network development, the ANN models were evaluated periodically with the validation data set to avoid making the model excessively specific to the original 65 cases; however, the validation data only maintained model generalization and were not used at any time to adjust the model coefficients. ANNs with processing layer elements numbering as high as 100 also were examined but were not systematically evaluated. ANN models without a processing layer and with all but a single input disabled evaluated the importance of the individual environmental variables.

\section{RESULTS}

Field-exposed plants that remained dry in the greenhouse had a $53 \%$ rate of infection, whereas $86 \%$ of the field-exposed plants that underwent a subsequent wet period were infected with tan spot (Table 1). Tan spot infection on field-exposed dry plants and plants with additional wetness corresponded, except for one case in 1995. Diseased plants left dry after field exposure corresponded to the detection of $P$. tritici-repentis spores by the Burkard spore sampler in 37 of 45 sampling periods with six missing data values.

TABLE 1. Tan spot incidence on wheat plants exposed for a 24-h period to a small wheat field environment in Fargo, ND

\begin{tabular}{lcccc}
\hline & \multicolumn{3}{c}{ Year } & \\
\cline { 2 - 3 } & 1993 & 1994 & 1995 & Total \\
\hline $\begin{array}{l}\text { No. of plants infected with tan } \\
\quad \begin{array}{l}\text { spot/no. of bioassays } \\
\quad\end{array}\end{array}$ & $22 / 44$ & $18 / 30$ & $11 / 23$ & $51 / 97$ \\
$\begin{array}{l}\text { No. of plants infected with tan } \\
\text { spot after an additional 24 h of } \\
\quad \text { wetness/no. of bioassays }\end{array}$ & $35 / 44$ & $29 / 30$ & $19 / 23$ & $83 / 97$ \\
$\begin{array}{l}\text { Conidia detected/no. of } \\
\text { bioassays }\end{array}$ & $27 / 40$ & $22 / 28$ & $21 / 23$ & $70 / 91$ \\
$\begin{array}{l}\text { Tan spot severity on flag leaves } \\
\quad(\%) \text { in wheat field }\end{array}$ & 7 & 20 & 14 & \\
$\begin{array}{l}\text { Disease severity on leaves } \\
\text { adjacent to flag (\%) }\end{array}$ & $\ldots$ & 56 & 47 & \\
\hline
\end{tabular}

a A Burkard spore sampler aided the evaluation of the bioassay. The Burkard spore collection data for 4 days in 1993 and 2 days in 1994 are missing due to mechanical problems.

${ }^{b}$ Foliar disease severity was measured in nearby plots. Leaves adjacent to the flag leaves were not evaluated for disease severity in 1993. 
TABLE 2. Accuracy of the statistical and optimal artificial neural network (ANN) models for prediction of tan spot infection periods on susceptible wheat plants exposed for a 24-h period to a wheat field environment in Fargo, ND

\begin{tabular}{|c|c|c|c|c|}
\hline & \multicolumn{2}{|c|}{$\begin{array}{l}\text { With wetness } \\
\text { sensor input }^{\mathrm{a}}\end{array}$} & \multicolumn{2}{|c|}{$\begin{array}{l}\text { Without wetness } \\
\text { sensor input }{ }^{\mathrm{a}}\end{array}$} \\
\hline & $\begin{array}{c}\text { Development } \\
(\%)\end{array}$ & $\begin{array}{c}\text { Validation } \\
(\%)\end{array}$ & $\begin{array}{c}\text { Development } \\
(\%)\end{array}$ & $\begin{array}{l}\text { Validation } \\
(\%)\end{array}$ \\
\hline $\begin{array}{l}\text { Multivariate } \\
\text { discriminant } \\
\text { regression }\end{array}$ & 91 & 50 & 86 & 55 \\
\hline $\begin{array}{l}\text { Stepwise logistic } \\
\text { regression } \\
\text { ANN }\end{array}$ & $\begin{array}{l}69 \\
99\end{array}$ & $\begin{array}{l}68 \\
87\end{array}$ & $\begin{array}{l}74 \\
99\end{array}$ & $\begin{array}{l}62 \\
81\end{array}$ \\
\hline
\end{tabular}

a Data consisted of a total of 97 exposure periods, of which 65 cases were used in model development and 32 were reserved for validation analysis. Flatplate resistance sensor data were withheld as model input to assess the impact on accuracy.

Control plants that remained dry were free of tan spot lesions, except for one period in 1994 when the control plant had two lesions and the field-exposed bioassay plant had 42 lesions; therefore, all cases were included in subsequent analyses (Table 1).

No relationship between a single independent variable and the dependent variable was obvious visually (Fig. 1). The highest linear correlation was -0.58 between $\mathrm{RH}$ and leaf-wetness resistance.

Even though multivariate discriminant analysis correctly distinguished more than $90 \%$ of tan spot infection periods in training data, correct prediction of validation data was equal only to chance (Table 2). The discriminant model without wetness-sensor inputs had a slightly increased prediction accuracy when based on the validation data but decreased an equal amount when based on the training data. Logistic regression correctly predicted fewer of the 65 training cases but more of the validation cases than did discriminant analysis (Table 2). The stepwise logistic model that included wetness-sensor data selected wetness, temperature, and cosine of wind direction as inputs. When wetness-sensor input was withheld from the logistic model, $\mathrm{RH}$ was the only input selected, and prediction accuracy for validation data decreased by $6 \%$.

The back-propagation ANN models with a hyperbolic tangent transfer function were the most accurate of the three techniques. The average prediction accuracy for 200 models with zero to nine PEs ranged from 74 to $77 \%$ on the validation data and converged on the data used for model development (Fig. 2). The best neural network model had five PEs and correctly predicted $99 \%$ of the training cases and $87 \%$ of the validation cases. ANN model performance on validation cases dropped $6 \%$ in accuracy when wetness-sensor input was withheld, but remained superior to the statistical models (Table 2). Models with other than one, five, or six intermediate PEs were similar in accuracy. ANN models with one PE had inferior accuracy compared to the other comprehensively tested models, and models with five and six PEs were optimal (Fig. 2). An examination of models with more than nine PEs or with different architectures failed to find any models with equal or superior performances to the model with five PEs.

Based on validation cases, the best ANN model with wetnesssensor inputs included was $100 \%$ accurate for periods when infection occurred and $73 \%$ accurate for periods when infection did not occur. Of the four noninfection cases missed by the ANN model, three were associated with high amounts of precipitation. Two of the rainy days had high average wind speeds of greater than $6.8 \mathrm{~m} \mathrm{~s}^{-1}$, relative humidities greater than $90 \%$, and nearly continuous wetness. When wetness data were withheld from the network development, six events were missed, two of which corresponded to the other four errors. Accuracy was $88 \%$ for tan spot infection periods and $73 \%$ for noninfection periods.

When the performance of ANN models with single input and no processing layer were evaluated, models with precipitation, $\mathrm{RH}$, wetness, or temperature predicted tan spot infection most accu-

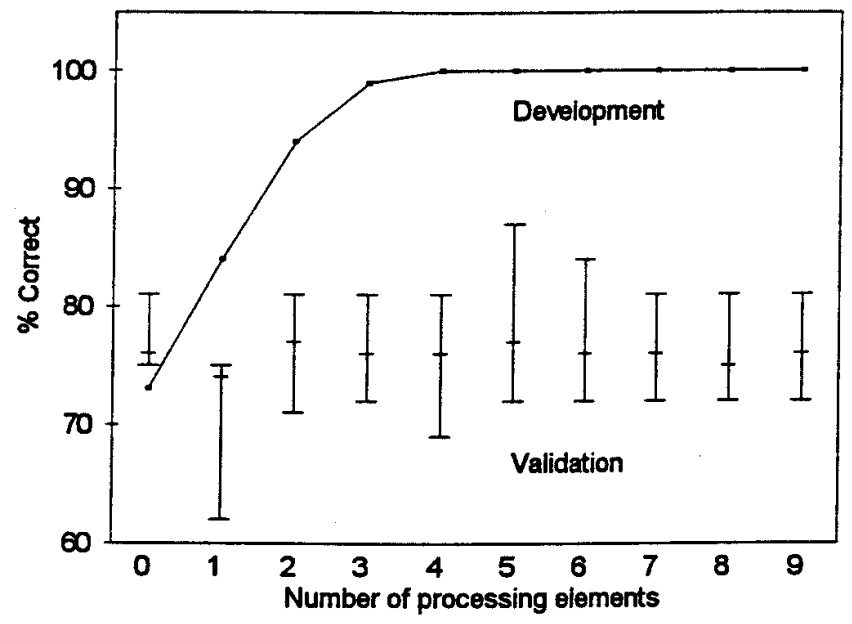

Fig. 2. Artificial neural network prediction accuracy for tan spot infection of wheat as a function of the number of elements in the processing layer. Each I bar consists of the range and mean validation accuracy for 20 neural networks with random initialization weights. The line consists of the maximum prediction accuracy of the 20 neural networks during model development.

rately (i.e., above the average for the set of nine inputs; Table 3). When growth stage, cosine of wind direction, wind speed, solar radiation, or sine of wind direction was analyzed singly, accuracy was less than $69 \%$.

\section{DISCUSSION}

The mobile bioassay with postexposure wet period was slightly more efficient in sampling inoculum of $P$. tritici-repentis than the Burkard sampler (86 versus $77 \%$ positives; Table 1 ). Nearly all exposure periods that resulted in infection without postexposure wetness were contained within the set wetted after exposure. Thus, we concluded that tan spot lesions on the former treatment were due to a favorable environment, inoculation, and infection during the 24-h exposure. Consequently, the mobile bioassay and automated weather station provided a reasonable assessment of tan spot infection periods.

Discriminant models fit the training data set well but performed too poorly on validation data to be of value for disease forecasting. Logistic models were better at generalization than discriminant models and predicted both data sets comparably. The stepwise process tended to select input variables for the logistic model that also were identified as important in a sensitivity analysis of the back-propagation neural network. Input variables (wetness, RH, and temperature) identified by both the logistic and ANN models probably represented essential requirements for infection by $P$. tritici-repentis (8).

Overparameterization and overfitting of data are risks in ANN model development, particularly when the network converges as in this study. Smith (17) recommends beginning network development at zero elements in the processing layer and increasing the number until the fit on the validation data set is maximized. Also, if the processing layer is smaller than the input layer information, compression must occur during processing. The software used in this study, NeuralWorks, can prevent overfitting of networks by periodically testing model accuracy on a second data set. Though effective for optimal model selection, this method compromises the comparison of ANNs with the statistical models, because specified conditions for validation differ. Even so, the best model was $99 \%$ accurate on the first data set (Table 2), and that model or a highly simialar ANN model may have been selected using the criterion of convergence. This model was nearly $20 \%$ more accurate than stepwise logistic regression both with and without leaf wetness-sensor inputs. Also, the entire population of ANN models with zero to nine PEs averaged 6 to $9 \%$ higher prediction accuracy 
than the stepwise logistic models, and only one ANN model had a prediction accuracy that was less than the logistic regression result (Fig. 2). This indicates that ANNs were superior to statistical models in prediction of tan spot infection.

The best ANN model had four false positives, two of which were associated with prolonged stormy weather, which may have been unfavorable for tan spot infection because conidia are readily liberated only from dry lesions. Windborne spores are susceptible to removal from the air mass by rain scrub, and any conidia on the bioassays could be either washed or blown from the leaves (5). The third false positive occurred during a period of intermittent precipitation, with a relatively cool temperature $\left(<16^{\circ} \mathrm{C}\right)$ and a high wetness-sensor resistance measurement, indicating that despite precipitation leaf wetness and temperature conditions may not have been favorable for tan spot infection. The fourth misidentified case occurred late in the growing season (Zadoks growth stage greater than 90), and although precipitation was absent, the wetness-sensor reading indicated that ample dew was present. Weather patterns during periods missed by the ANN that lacked wetness-sensor input were not interpreted as easily.

ANN models share a limitation with all empirical methods in that prediction accuracy is valid mainly within the range of the independent variables on which the models are developed (1). The assay system provided 97 discrete episodes of outdoor environment for analysis, which appeared to be adequate for this study but which were thought to be too few to allow reservation of data for additional validation. More cases would be needed for a model that would operate reliably under a wider range of weather conditions; however, interregional variability may compromise model accuracy on a local level.

ANN accuracy appears to be acceptable for use as forecasters in production agriculture, but field testing of models is an essential part of any development effort. ANN models can be deployed by means of a ' $C$ ' code callable function. The resulting program, combined with any code that might be required to transform raw data and interface with users, could be compiled to form a forecasting module for field operation. Multiple disease or pest forecasts are possible by adding more PEs to the ANN output layer or by having additional models in the same forecasting module; however, separate models likely would reduce the cost of system maintenance and enhancement (14).

The accuracy achieved in forecasting tan spot infection combined with the deployment ease of ANN models clearly demonstrates the applicability of ANN technology to plant disease forecasting. In the future, ANN models may provide nearly real-time disease forecasts and be applicable in other areas of plant pathology.

\section{ACKNOWLEDGMENTS}

Contribution from the North Dakota State University Agriculture Experiment Station, Fargo.

\section{LITERATURE CITED}

1. Campbell, C. L., and Madden, L. V. 1990. Introduction to Plant Disease Epidemiology. Wiley Interscience, New York.

2. Cook, D. F., and Wolfe, M. L. 1991. A back-propagation neural network to predict average air temperature. Artif. Intel. Appl. 17:1482-1487.

3. Cook, R. J., and Veseth, R. J. 1991. Wheat Health Management. The American Phytopathological Society, St. Paul, MN.
TABLE 3. Single-variable artificial neural network models were evaluated for their ability to predict tan spot infection caused by Pyrenophora triticirepentis on wheat bioassay plants exposed for a 24 -h period to a small wheat field environment in Fargo, ND

\begin{tabular}{lcc}
\hline Variable & $\begin{array}{c}\text { Development } \\
\text { data set }(\%)^{\mathrm{a}}\end{array}$ & $\begin{array}{c}\text { Validation } \\
\text { data set }(\%)\end{array}$ \\
\hline Precipitation $(\mathrm{mm})$ & 83 & 69 \\
Proportional relative humidity & 72 & 65 \\
$\log _{10}$ (wetness sensor resistance) $(\mathrm{k} \Omega)$ & 71 & 75 \\
Temperature $(\mathrm{C})$ & 66 & 72 \\
Zadoks growth stage & 54 & 65 \\
Cosine wind direction & 52 & 66 \\
$\log _{10}$ (wind speed) $\left(\mathrm{m} \mathrm{s}^{-1}\right)$ & 51 & 69 \\
$\log _{10}$ (solar radiation) $\left(\mathrm{kJ} \mathrm{m}^{-2} \mathrm{~s}^{-1}\right)$ & 47 & 62 \\
Sine wind direction & 43 & 62 \\
\hline
\end{tabular}

${ }^{a}$ Evaluation of a 65 -case data set used in model construction and a 32-case data set in which maximum accuracy was the criterion to halt iterative processing.

4. Elizondo, D., Hoogenboom, G., and McClendon, R. W. 1994. Development of a neural network model to predict daily solar radiation. Agric. For. Meteorol. 71:115-132.

5. Francl, L. J. 1995. Challenge of bioassay plants in a monitored outdoor environment. Can. J. Plant Pathol. 17:138-143.

6. Francl, L. J., Jordahl, J. G., and De Wolf, E. D. 1995. Conidiation of Pyrenophora tritici-repentis on wheat leaves. (Abstr.) Phytopathology 85:1044.

7. Francl, L. J., Panigrahi, S., and Pahdi, T. 1995. Neural network models that predict leaf wetness. (Abstr.) Phytopathology 85:1182.

8. Hosford, R. M., Jr., Larez, C. R., and Hammond, J. J. 1987. Interaction of wet period and temperature on Pyrenophora tritici-repentis infection and development in wheats of differing resistance. Phytopathology 77 : 1021-1027.

9. Hosmer, D. W., Jr., and Lemeshow, S. 1989. Applied Logistic Regression. Wiley Interscience, New York.

10. Kohli, M. M., Mehta, Y. R., and de Ackermann, M. D. 1992. Spread of tan spot in the southern cone region of South America. Pages. 86-90 in: Advances in Tan Spot Research. L. J. Francl, J. M. Krupinsky, and M. P. McMullen, eds. North Dakota State University Agricultural Experiment Station, Fargo.

11. McMullen, M. P., and Nelson, D. R. 1992. Tan spot and five years of wheat disease survey. Pages 80-85 in: Advances in Tan Spot Research. L. J. Francl, J. M. Krupinsky, and M. P. McMullen, eds. North Dakota State University Agricultural Experiment Station, Fargo.

12. Murase, H., Nishiura, Y., and Honami, N. 1994. Textural features/neural network for plant growth monitoring. Am. Soc. Agric. Eng. Paper 944016.

13. Rees, R. G., and Platz, G. J. 1980. The epidemiology of yellow spot of wheat in southern Queensland. Aust. J. Agric. Res. 31:259-267.

14. Ritchie, J. T. 1989. Crop models and decision making: Scenario for the future. Pages 106-117 in: Climate and Agriculture. A. Weiss, ed. American Meteorology Society, Charleston, SC.

15. Shearer, B. L., and Zadoks, J. C. 1972. The latent period of Septoria nodorum in wheat. 1 . The effect of temperature and moisture treatments under controlled conditions. Neth. J. Plant Pathol. 78:231-241.

16. Shearer, B. L., and Zadoks, J. C. 1974. The latent period of Septoria nodorum in wheat. 2 . The effect of temperature and moisture under field conditions. Neth. J. Plant Pathol. 80:48-60.

17. Smith, M. 1993. Neural Networks for Statistical Modeling. International Thomson Computer Press, New York.

18. Thai, C. N., and Shewfelt, R. L. 1991. Modeling sensory color quality of tomato and peach: Neural networks and statistical regression. Trans. ASAE 34:950-955.

19. Wasserman, P. D. 1993. Advanced Methods in Neural Computing. Van Nostrand Reinhold, New York.

20. Yang, X. B., Batchleor, W. D., and Tschanz, A. T. 1995. A neural network model to predict soybean rust. (Abstr.) Phytopathology 85:1172.

21. Zadoks, J. C., Chang, T. T., and Konzak, C. F. 1974. A decimal code for the growth stage of cereals. Weed Res. 14:415-421. 